\title{
Post-processing of 3D printed metallic micro-parts
}

\author{
Rafaël Brunet, Lucas A. Hof \\ Mechanical Engineering, École de Technologie Supérieure (ÉTS), Montréal, Canada \\ rafael.brunet.1@ens.etsmtl.ca \\ lucas.hof@etsmtl.ca
}

\begin{abstract}
The fourth industrial revolution is transforming current manufacturing approaches. One of the main objectives of this Industry 4.0 is to mass produce highly customized products. Hence, this requires new types of production methods to manufacture parts with a large variety of dimensions and geometry.

The present research project aims to contribute to this objective in collaboration with an industrial partner. They are a solution provider in the high-precision metal additive manufacturing (AM) field, combining AM, nanotechnology and machine learning providing unique freedom of design and material choice for high-precision parts by their developed 3D printer combining deposition of nanoparticle monolayers with laser sintering. However, most of their generated micro metal parts require post-processing steps before entering industrial applications. As additional process steps appear to be undesired, deploying post-processing could have an additional benefit of reducing the overall manufacturing time by balancing layer height and the polishing process to reduce the surface roughness of the generated metal micro-parts. Among other methods, electrochemical polishing (EP) seems a promising candidate for post-processing such parts. Nevertheless, EP is still unexplored in combination with this novel high-precision 3D printing method.
\end{abstract}

This project aims to find a trade-off between layer thickness, printing speed and polishing. An optimum should be achieved to get smooth and flat surfaces to maintain high quality parts at reasonable fabrication time. Secondary, the project aims to acquire more knowledge of the printing technology and its interaction with post-processes on various alloys.

Handling of these microparts, such as manual operations to attach electrodes for EP, is extremely challenging because of their small size (sub-mm) and relative brittleness.

EP is the reverse of electroplating; hence, it dissolves metal on the surface of the part resulting in a smooth surface. This method does not apply forces on the brittle parts, requires very little handling and is able the reach very low surface roughness (submicron) on most geometries increasing fatigue resistance of the parts.

However, EP of small micro-parts encounters some challenges. It requires the polishing medium (electrolyte) to be in contact with the part surface and a mechanical contact is required to close the electrical circuit (current flowing to the part). As generating a contact with typical metal parts is trivial, this is an issue for the printed micro-parts as contact points are relatively large compared to the parts to be polished. Second, EP produces gases that can interfere with medium contact of the part.

This study presents a novel prototype for an EP setup to overcome such issues for polishing metallic micro-parts. It consists of a rotating drum designed to evacuate gases and vary while being mostly constant the contact point by tumbling the parts in a conductive drum. This ensures uniform and smooth surface roughness reduction on parts of all sizes and shapes. A preliminary study of the prototype impact shows that the printing time could be reduced by four times.

Further, ongoing tests of EP on different alloys such as SS316, Ti and CuSn will be presented. 